DOI: $10.20472 / E S .2015 .4 .2 .002$

\title{
DOES BITCOIN FOLLOW THE HYPOTHESIS OF EFFICIENT MARKET?
}

\section{JAKUB BARTOS}

\begin{abstract}
:
Bitcoin has emerged as phenomenon of the financial markets as the currency without any central authority. Recent events of Bitcoin has risen question about its behavior and there is crucial question if the price of Bitcoin follows hypothesis of efficient markets. In this paper, there are introduced the main features of Bitcoin and analyzed its price behavior. We found out that price of the most famous cryptocurrency Bitcoin follows the hypothesis of efficient markets and it immediately react on publicly announce information. Furthermore, Bitcoin can be seen as standard economic good that is priced by interaction of supply and demand on the market. These factors can be driven by macro financial development or by speculative investors, but there weren't found any significant impact of these factors on price of Bitcoin.
\end{abstract}

\section{Keywords:}

Cryptocurrencie, Bitcoin, Financial market

JEL Classification: G14, G02, C22

\section{Authors:}

JAKUB BARTOS, University of Economics, Prague, Czech Republic, Email: kbartos11@gmail.com

\section{Citation:}

JAKUB BARTOS (2015). Does Bitcoin follow the hypothesis of efficient market?. International Journal of Economic Sciences, Vol. IV(2), pp. 10-23., 10.20472/ES.2015.4.2.002 


\section{Introduction}

Innovations are important contributors of economic development and it brings new solutions to market, but it could face some problems in the first stage of development. Financial innovations were run mostly by institutions (Lerner, 2003) but introducing of the internet has completely changed the way real economy works. All Internet users can interact at once and sharing and exchanging information is almost cost free (Kristoufek, 2013). Internet-based electronic marketplaces leverage information technology to match buyers and sellers with effectiveness and lower transaction costs, but there have been still intermediary institutions causing some transaction costs (Bakos, 1998).

Digital currencies propose a shift away from established design of financial system infrastructures. Information systems and technological solutions like peer-to-peer connectivity and cryptographic algorithms allow for decentralized organization, operational security and transparency, thus opposing the centrally coordinated and less transparent traditional monetary systems' structures (Glaser, et al., 2014). Era of the Internet, financial innovation and other development have emerged alternative currency that is exclusively electronic and based on peer-to-peer mechanism, which allows near real time transactions, transparent creation by open algorithm and store of history of transactions. On the other hand, cryptocurrencies face the problem of money laundering and trafficking of illegal substances of various kinds (Brezo \& Bringas, 2012).

Cryptocurrencies are used as financial instruments and especially Bitcoin is called alternative investment with diversification benefits (Briére, et al., 2013). The most popular cryptocurrencies are Bitcoin, Litecoin, Dogecoin and Ripple, but there are hundreds of different cryptocurrencies on the market. In this paper will be analyzed Bitcoin as the most popular cryptocurrency. Previous studies tried to determine the key factors driving the price of Bitcoin and there were found that price is affected by supplydemand interaction of Bitcoin, Bitcoin's attractiveness for investors and global macroeconomic and financial developments (Kristoufek, 2013; Buchholz, et al., 2012; van Wijk, 2013). On the other hand, Ciaian et al. (2014) do not support previous findings that the macro-financial developments are driving Bitcoin price.

This paper estimates the impact of publicly announced information on price of Bitcoin and therefore if price of Bitcoin reflects the hypothesis of efficient market (Fama, 1970). Efficient markets are when in any given time, the prices on the market already reflect all known information and change fast to reflect new information. Therefore no one could outperform the market by using the same information that is already available to all investors, except through luck. According to theory of efficient markets investors decide rationally, but behavioral finance claim that investors are influenced by many factors and their decision making could be irrational. There are many observed market movements that are not explained by the arguments of efficient market hypothesis. Such market movements are called anomalies. French (1979) found out significant negative return of stocks on Monday. Singal (2006) explains the effect as tendency of companies to release bad news on Friday after market close that leads to decrease 
stock prices on Monday. There are more market anomalies such as January effect, underpricing of IPO, the size effect, the neglected firms' effect, the merger and acquisition effect and the low P/E ratio effect. There are anomalies on the markets which partly deny existence of efficient markets. Theory of behavioral finance is still progressing and it has been discussed in recent years.

There are introduced the main features of Bitcoin and the econometric models are developed to estimate the determinants of Bitcoin price. Especially, it evaluates the impact of events on price of Bitcoin and there were found out, that price of Bitcoin follows hypothesis of efficient markets. The results are discussed in the last part and there are suggested improvements of this paper.

\section{Bitcoin}

Bitcoins are a digital, or virtual, currency that uses peer-to-peer technology to facilitate instant payments. Since Bitcoins are not a fiat currency (currency that a government has declared to be legal tender, but is not backed by a physical commodity), they are not controlled by a single entity like a central bank and are therefore sometimes referred to as a decentralized currency (Maggi, 2014). Bitcoin attempts to overcome the weaknesses of both fiat and gold-based money, functioning as an algorithmic currency with a deterministic supply and growth rate tied to the rigor of mathematics. No government or other central authority can manipulate the supply of Bitcoins (Yermack, 2013). Acceptance of payments with Bitcoin is growing, but still limited. Furthermore, regulators and governments start to create more regulation and structure to legitimize it as a currency and there is still ongoing discussion about whether Bitcoin is primarily an alternative currency or just speculative asset (European Central Bank, 2012).

Money creation in Bitcoin system is transparently realized by a distributed and open algorithm. The infrastructure allows near-real-time transaction monitoring via the public peer-to-peer network and the entire transaction history is stored in a chain of transactions (Nakamoto, 2008). Every unit of Bitcoin has own address with the public and private key. There is strong dependence between these keys and public key can be derived from the private key. Transaction is preceded with the private key and when it corresponds with the public key then transaction is confirmed. At any moment in time, there is a fixed number of Bitcoins and the block chain allows a user to prove ownership of a particular Bitcoin and to verifiably transfer ownership without the need for a single trusted third party (Brito, et al., 2014). The Blockchain represents all verified and valid transactions between users of the network (Glaser, et al., 2014). User can gain Bitcoins by accepting of transaction from another user or by mining.

Instead of being made on a printing press or by a central authority, Bitcoins are generated by solving complicated algorithmic searches by powerful computers, a process known as mining (Chowdbury, 2014). It is a process that involves programmers solving complex math problems with the computers in the network. The total number of Bitcoins that will be issued is capped at 21 million and it ensures the stable and well 
known supply of Bitcoins. This limit is expected to be reached in the year 2140 based on the way Bitcoins are created (Maggi, 2014).

Bitcoin's invention is revolutionary because for the first time the double spending problem can be solved without need for third party. Bitcoin does this by distributing the necessary ledger among all the users of the system via a peer-to-peer network. Every transaction is registered in the block chain and it ensures that the same Bitcoins have not been previously spent, thus it eliminates the double spending problem (Brito, et al., 2014). The block chain is the crucial feature to maintain the transparency of Bitcoin system, because all transactions are recorded there and double spending is impossible. Network of Bitcoin users is connected via digital wallets. It is software that generates addresses, keys and provides transactions. Bitcoins are stored in these wallets which exist either in the cloud or on a user's computer. Except mining you can get Bitcoin on many exchanges. You can change any currency for Bitcoin or vice versa. Furthermore, you can pay in some shops with Bitcoins for goods, mostly online.

\section{Analysis of Bitcoin}

\subsection{Theoretical model}

From previous analysis we can see that prices of cryptocurrencies have quite unusual behavior. Specially, Bitcoin started the year 2013 at level of \$US 13 per Bitcoin and it raised to $\$$ US 213 on $9^{\text {th }}$ April 2013, that could create potential profit of $1500 \%$ in less than four months. Afterwards, price of Bitcoin rocketed even higher to \$US 1132 on $29^{\text {th }}$ November 2013, that created potential profit $8600 \%$ since the beginning of 2013 . Additionally, there was a huge drop in prices and price of Bitcoin fell down to US $\$ 400$ on $11^{\text {th }}$ April 2014 and then price started to grow again and then fall to $\$ U S 350$ on $7^{\text {th }}$ November 2014.

Currencies can be seen as standard economic goods which are priced by interaction of supply and demand on the market, which are driven by macroeconomic variables of an issuing country or institution such as GDP, interest rates, inflation, unemployment and others. There are no macroeconomic fundamentals for digital currencies, the supply function either fixed (if currency amount is fixed) or it evolves according to some publicly known algorithm, which is the case of Bitcoin market and Litecoin as well (Kristoufek, 2013). As we know that supply will be fixed when amount of Bitcoins reach 21 million, but till that moment supply is driven by miners and numbers of new Bitcoins. On the other hand, the demand side of the market is not driven by macroeconomic development of particular country, but there are speculative investors who trade with the investment instruments. Most Bitcoin users do not mine, but purchase or trade for their Bitcoin. Mining doesn't affect average user much, but is still a very important part of Bitcoin ecosystem. Miners are motivated by reward of 50 Bitcoins for new formed block and generate new Bitcoins (Chowdbury, 2014). Important determinants of Bitcoin price are interaction between supply and demand of Bitcoin and it is outcome of interaction between supply and demand (Buchholz, et al., 2012). Search queries provided by Google Trends and Wikipedia have proved to be a useful source of 
information in financial applications ranging from the home bias and the traded volume explanations through the earnings announcements to the portfolio diversification and the trading strategies and it can be a good measure of interest in the currency (Kristoufek, 2013).

The role of global financial development, captured for example by stock exchange indices, exchange rates and oil prices have effect on price of Bitcoin (van Wijk, 2013). Market indexes may reflect the general macroeconomic and financial developments of the global economy, because it basically represents the performance of the biggest companies that influence the global economy. Previous studies found out that Bitcoin price is determined by three key factors such as supply-demand interaction of Bitcoin, Bitcoin's attractiveness for investors and global macroeconomic and financial developments (Kristoufek, 2013; Buchholz, et al., 2012; van Wijk, 2013). On the other hand, Ciaian et al. (2014) do not support previous findings that the macro-financial developments are driving Bitcoin price.

This model wants to evaluate previous findings on the current data, test the effect of the information on the price of Bitcoin and confirm the hypothesis of efficient markets that new available information are reflected in price of Bitcoin.

\subsection{Data}

Firstly, we got daily data of aggregated Bitcoin price index from multiple exchanges providing a weighted average Bitcoin and Litecoin price in USD, historical total number of Bitcoins which have been mined, total number of unique Bitcoin transaction per day and number of unique Bitcoin addresses used per day from 4.3.2013 to 31.7.2014 from Quandle. These data except price of Litecoin are used to analyze the supply of Bitcoin and test the effect on price.

Secondly, we got daily data of prices of financial instruments such as S\&P 500, Google, Facebook, Dow Jones and Gold from 4.3.2013 to 31.7.2014 from Finance Yahoo. We have chosen market indexes which show the performance of economic development and stock prices of innovative companies as Google and Facebook to try to explain the possible relationship.

Thirdly, we gained daily data of search queries for words "Bitcoin" from Wikipedia as proxies of demand for Bitcoin. The daily series of Wikipedia entries provide detailed picture of the behavior of Internet users' interest and attention. Then, we used data from press and articles about Bitcoin. We created a variable that describes positive and negative information about Bitcoin. We have variable with 8 positive events and variable with 6 negative events. Those events include announcements of acceptance of Bitcoin, establishment of exchanges, failures of exchanges and marketplaces, establishment of first ATM and ban from central authorities.

Finally, we had to adjust data because of some missing values. Market indexes and financial instruments are not traded during the weekends and public holidays so we had to omit those days and assign to the same dates of cryptocurrencies and interest rates to financial data. We got 349 observations for analysis of price of Bitcoin. 


\subsection{Data transformation and descriptive statistics}

Dataset concerns mostly variables that are based on daily financial data, which are mostly positive non stationary variables. Basically, variables as number of mined Bitcoins, total number of transaction, number of unique used addresses and visibility of Bitcoin on Wikipedia are times series as well and we have to transform those variables into their log forms so that the first differences are interpretable as growth rates.

We want to identify if variable is stationary and can be used in the regression analysis and ADF test will be used. The ADF test can be improved that it takes in account constant and trend term. The equations for those two tests are

$$
\begin{gathered}
\Delta y_{t}=\alpha+\theta y_{t-1}+v_{t} \\
\Delta y_{t}=\alpha+\theta y_{t-1}+\delta t+v_{t}
\end{gathered}
$$

where $\alpha$ represents constant term that means time series would be stationary around upward or downward trend and $y_{t}$ is dependent variable and $v_{t}$ is the error term. The null and alternative hypotheses are $H_{0}: \theta=0, H_{a}=\theta<1$ respectively, the null hypothesis implying non stationarity and the alternative hypothesis implying stationarity. Trending term $\delta t$ takes in account the possibility that the change in $y_{t}$ has certain change over time (Griffiths, et al., 2012). All variables are tested for stationarity using Augmented Dickey Fuller test. We found out that all variables are non-stationary. The first difference deal with non-stationarity and we have used first logarithmic difference of all variables.

We have made descriptive statistics of financial instruments and cryptocurrencies in the observed period. As we can in the table cryptocurrencies have higher return but much higher volatility than stocks and market indexes. Mean daily return of Bitcoin is $0,667 \%$ and Litcoin has even higher mean daily return $1,158 \%$. Standard deviation of Bitcoin is $11,5 \%$ and standard deviation of Litecoin is $8,5 \%$ that is pretty high comparing to another financial instruments as stocks of Google has mean daily return $0,1 \%$ and standard deviation $1,422 \%$ and market index as S\&P 500 has daily return $0,07 \%$ and standard deviation is $0,69 \%$. 
Table 1: Descriptive statistics of financial instruments (source: own calculation)

\begin{tabular}{c|ccccccc} 
& Bitcoin & Litecoin & Google & Facebook & DowJones & S\&P500 & Gold \\
\hline $\begin{array}{c}\text { Mean } \\
\text { return }\end{array}$ & $0,66 \%$ & $1,158 \%$ & $0,104 \%$ & $0,314 \%$ & $0,0475 \%$ & $0,069 \%$ & $-0,05 \%$ \\
$\begin{array}{c}\text { Standart } \\
\text { deviation }\end{array}$ & $11,44 \%$ & $8,552 \%$ & $1,422 \%$ & $2,851 \%$ & $0,646 \%$ & $0,690 \%$ & $1,152 \%$ \\
Maximum & $40,14 \%$ & $58,852 \%$ & $13,79 \%$ & $29,611 \%$ & $2,183 \%$ & $2,183 \%$ & $4,905 \%$ \\
Minimum & $-34,41 \%$ & $-35,95 \%$ & $-4,66 \%$ & $-6,950 \%$ & $-2,342 \%$ & $-2,50 \%$ & $-8,52 \%$
\end{tabular}

Correlation matrix of financial instruments and cryptocurrencies shows us, that Bitcoin has correlation close to 0 with other securities and with Litecoin as well. It can be first sign that it can be used in portfolio management as Chowdbury (2014) shows that well diversified portfolios with a small Bitcoin component $(2 \%)$ improve the performance of risk-return trade off. On the other hand, there were high correlation between price of Bitcoin and total number of Bitcoins which have been mined, total number of unique Bitcoin transaction per day and number of unique Bitcoin addresses used per day, but logarithmic differences rapidly decreased correlation among variables and moreover the log-log specification allows for an easy interpretation of the relationship as the elasticity.

Table 2: Correlation matrix of financial instruments (source: own calculation)

\begin{tabular}{lcccccc|c} 
Gold & S\&P500 & Facebook & $\begin{array}{c}\text { Dow } \\
\text { Jones }\end{array}$ & Google & Litecoin & Bitcoin & \\
\hline 1.0000 & 0.0048 & 0.0583 & -0.0210 & -0.0407 & 0.0449 & -0.0262 & Gold \\
& 1.0000 & 0.3608 & 0.9570 & 0.5733 & 0.1056 & -0.0574 & S\&P500 \\
& 1.0000 & 0.2940 & 0.3303 & 0.0120 & -0.0462 & Facebook \\
& & 1.0000 & 0.5024 & 0.1086 & -0.0473 & $\begin{array}{c}\text { Dow } \\
\text { Jones } \\
\text { Google }\end{array}$ \\
& & & 1.0000 & 0.0533 & -0.0059 & Litecoin \\
& & & & 1.0000 & 0.0453 & & Bitcoin
\end{tabular}




\subsection{Methodology}

Ordinary Least Squares estimates linear relations between variables and it finds the best possible linear relationship between the dependent and independent variables. It has some assumptions and we should test heteroskedasticity, correlation and cointegration. Basically, heteroskedasticity is fixed by logarithmic transformation of all variables.

Correlation concerns correlation between the error terms of subsequent time periods and it should be zero. It can be tested with the correlogram of the residuals of regression analysis or Breusch-Godfrey LM test could be useful to validate this assumption.

If residuals of regression with all non-stationary variables are stationary, then it can be assumed that there is cointegration between dependent and independent variables. To test this assumption, residuals of the regression which include all non-stationary variables will be tested for stationary using ADF test (van Wijk, 2013).

Almost all variables have been transformed to first difference and it can cause the loss of information in OLS model. First difference of variables will result in loss the long run effect in OLS, but Error Correction Model (ECM) allows to model short run and long run affects. Furthermore, ECM solves problem with cointegration, because it includes stationary and non stationary variables as well. ECM is built from autoregressive distributed lag model that is here:

$$
y_{t}=\alpha+\theta_{1} y_{t-1}+\beta_{0} x_{t}+\beta_{1} x_{t-1}+v_{t}
$$

Following linear transformation is called the Bårdsen (1989) transformation and it has this steps:

$$
y_{t}=\alpha+\left(\theta_{1}\right) y_{t-1}+\beta_{0}\left(\Delta x_{t}\right)+\left(\beta_{0}+\beta_{1}\right) x_{t-1}+v_{t}
$$

We can use Ordinary Least Squares to estimate previous equation.

Long run effect is derived from autoregressive distributed lag model and there can be assumed that $y_{t}=y_{t-1}=y, x_{t}=x_{t-1}=x$ and $v_{t}=0$. We use this assumption and transform it to the following equation:

$$
y\left(1-\theta_{1}\right)=\alpha+\left(\beta_{0}+\beta_{1}\right) x
$$

The long run effect of the ECM model follows:

$$
y=\frac{\propto}{1-\theta_{1}}+\frac{\left(\beta_{0}+\beta_{1}\right) x}{1-\theta_{1}}
$$

Short run effect consists $\beta_{0}\left(\Delta x_{t}\right)$ and $v_{t}$ (Griffiths, et al., 2012).

Error Correction Model is dynamic model, because it corrects the short run changes with long run trend that errors do not deviate far from the long run trend. ECM model could be better estimator than OLS when we use time series data. 


\subsection{ECM results}

In order to examine the impact of variables on price of Bitcoin we have run regression with price of Bitcoin as dependent variable and other factors various factors as independent variables. Firstly, we run regression with all independent variables which were described in theoretical part such as daily prices of market indexes S\&P 500 and Dow Jones, daily prices of stocks Google and Facebook, prices of gold and Litecoin, number of total number mined Bitcoins, total number of transactions of Bitcoin, number of unique used addresses of Bitcoin, visibility of Bitcoin on Wikipedia and finally created variable positive and negative events which describes released information.

Secondly, we run separated regression for three key factors that determine price of Bitcoin according to theoretical model such as supply-demand interaction of Bitcoin (model 3), Bitcoin's attractiveness for investors with public released information (model 4) and global macroeconomic and financial developments (model 2).

Finally, we run model without financial data, because it was explained that in previous section that macroeconomics and financial development don't affect price of Bitcoin (model 5).

Error Correction Model solves the problem of cointegration and distinguish long run and short run effects as there is added first order autoregressive term and first order autoregressive term of the price of Bitcoin.

Table 3: Model 5 with dependent variable Id_USDBitcoin

\begin{tabular}{|c|c|c|c|c|c|}
\hline & (1) & (2) & (3) & (4) & (5) \\
\hline \multirow[t]{2}{*}{ const } & -0.01455 & 0.007147 & $-0.02091^{*}$ & $0.007996^{*}$ & -0.01499 \\
\hline & $(0.01319)$ & $(0.004698)$ & $(0.01235)$ & $(0.004561)$ & $(0.01298)$ \\
\hline \multirow[t]{2}{*}{ Id_GoldUSD } & 0.2436 & 0.3724 & & & \\
\hline & $(0.3898)$ & $(0.4038)$ & & & \\
\hline \multirow[t]{2}{*}{ Id_GoldUSD_1 } & 0.5188 & 0.5503 & & & \\
\hline & $(0.3870)$ & $(0.3991)$ & & & \\
\hline \multirow[t]{2}{*}{ Id_S_P500 } & 2.834 & 1.011 & & & \\
\hline & $(2.458)$ & $(2.536)$ & & & \\
\hline \multirow[t]{2}{*}{ Id_S_P500_1 } & -3.503 & -2.784 & & & \\
\hline & $(2.492)$ & $(2.575)$ & & & \\
\hline \multirow[t]{2}{*}{ Id_Facebook } & -0.06133 & -0.04658 & & & \\
\hline & $(0.1798)$ & $(0.1875)$ & & & \\
\hline \multirow[t]{2}{*}{ Id_Facebook_1 } & 0.04058 & 0.07066 & & & \\
\hline & $(0.1800)$ & $(0.1869)$ & & & \\
\hline \multirow[t]{2}{*}{ Id_DJ } & -1.318 & 0.2344 & & & \\
\hline & $(2.462)$ & $(2.538)$ & & & \\
\hline \multirow[t]{2}{*}{ Id_DJ_1 } & 3.486 & 2.842 & & & \\
\hline & $(2.462)$ & $(2.558)$ & & & \\
\hline
\end{tabular}




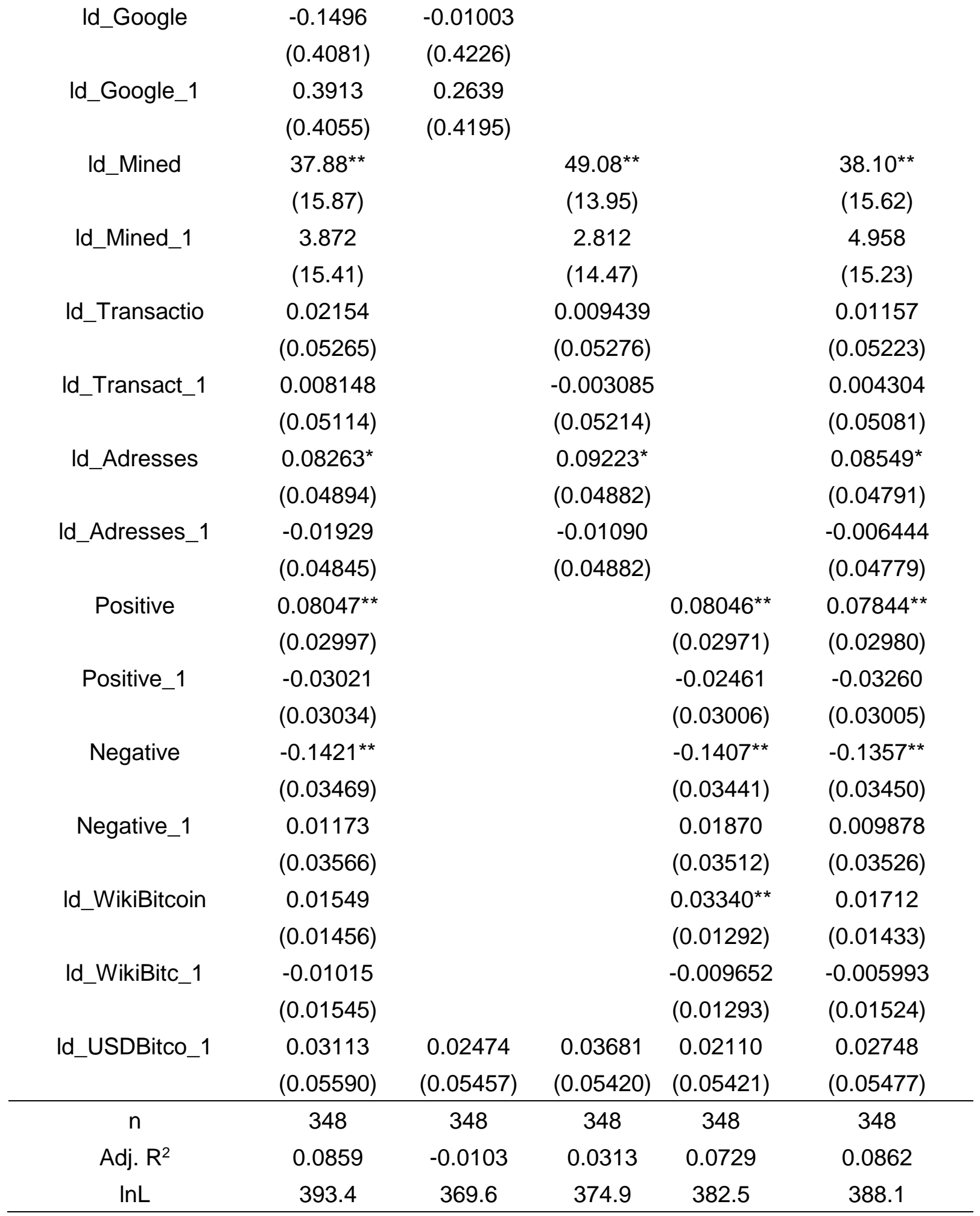

${ }^{*}$ Indicates significance at the $10 \%$ level, ${ }^{* *}$ at $5 \%$ level, and ${ }^{* * *}$ at the $1 \%$ level Regression analysis using 348 observations

We have also analyzed F-test of the sum squared residuals of all models and we can validate if full model explains more than another models. The sum of squared residuals is lowest in model with all variables and therefore according to F-statistics it is not 
appropriate to delete variables from regression. For the further tests we will consider full model.

Cointegration and heterskedasticity is solved by model and logarithmic transformation. We use Breusch Godfrey LM with one lag to test the serial correlation of the model. The results that are obtained doesn't reject null hypothesis that there is no serial correlation of the term error. $F$ statistics with $p$-value 0,32 conclude that there is no serial correlation in the error term.

Our regression analysis didn't show any trend in the long run. Any of the variables are significant and there is no effect on price of Bitcoin in long run. In the other words, there is no impact of lags variables on price of Bitcoin. Price of Bitcoin is independent to all of market indicators and we cannot explain price of Bitcoin according to development of financial instruments. Furthermore, we can conclude that there weren't any insider trading, because there is no significant impact of lags of positive or negative events and information is transformed to the day of event not before.

From the regression conclusion can be drawn that the change in the price of Bitcoin in the short run depends on change in number of total mined Bitcoins, change in number of unique used addresses per day and if positive or negative event happened. The rest of variables are insignificant, but they improve explanatory power of model.

As we can see from results that $1 \%$ increase in number of mined Bitcoins lead to $3788 \%$ increase in price of Bitcoin. This result could look ridiculous but time series started at almost 11 million mined Bitcoins and ended up at 13 million. The change was 2223725 of mined Bitcoin what is around $20 \%$ change. Price of Bitcoin started at US\$ 35 and ended up US\$ 572 what is $1521 \%$ increase in price. We could consider opposite causality between these 2 variables, because miners are rewarded with Bitcoins, but we have found out very low correlation and therefore this variable has explanatory power of price of Bitcoin.

In addition, $1 \%$ increase in number of unique Bitcoin addresses used to transaction leads to $8,2 \%$ increase in price of Bitcoin. It can signalize the importance the supply and demand interaction such as it describes more likely the usage of Bitcoin and we can see as Bitcoin gets more popular there is increase in price and number of used unique addresses could be good indicator.

Finally, we tested the impact of positive and negative announcements. Days where positive events were announced, there is $8 \%$ higher price of Bitcoin that another days and days where negative events were announced, there is $14,21 \%$ lower price of Bitcoin that another days. It could seem that it is connected with evolution of price of Bitcoin, but events are distributed through whole period and there are not only in upward or downward trend in period where events are located. As we can see markets of cryptocurrencies react significantly on fpublic announcements and it confirms hypothesis of efficient markets. 


\subsection{Discussion of models}

There are some restrictions and weaknesses of the model that had to be done in this paper. Firstly, there is very limited time and time series are quite short due to Bitcoin is new instrument and it could conclude to unsatisfying results. Secondly, we had to omit some data due to stocks and market indexes are not traded during weekend and it could harm the explanatory power of model. Thirdly, there were used limited number of market and financial indicators, because it is not clear that indicators could impact price of Bitcoin. There could be used different kinds of variables, that describe better financial and economic development, but there is a problem of limited daily data available. Anyway, models follow previous findings of researches that financial and economic development don't drive price of Bitcoin (Ciaian, et al., 2014).

Furthermore, analysis of price of Bitcoin shows that price is driven by supply and demand interaction as number of mined Bitcoins and numbers of unique used Bitcoins are significant factors. It exactly describes supply and demand interaction, because it can be considered that number of mined Bitcoins is on supply side and number of unique addresses is on demand side. This conclusion follows previous results of Buchholz et al. (2012). On the other hand, Kristoufek (2013) found out that price of Bitcoin could be explained by search queries, that symbolize interest in currency and price is driven by speculative investors. However, there were analyzed data only till 30.6.2013 and price in this period had mostly upward trend and price could be explained by trading of speculative investors. Afterwards, there were more changes in prices of Bitcoin such as there were upward trend and downward trend as well in the time series. Results show that variable search queries from Wikipedia doesn't have any significant effect on price of Bitcoin. On the other hand, there were used data only from Wikipedia and it doesn't have to explain interest of investors, because they could search more likely on Google and usage of data from Google could improve our results.

Finally, paper analyzed the effect of announced events on price of Bitcoin. There were found that positive events have positive significant impact on price of Bitcoin at the day of event and negative events have negative significant effect on price of Bitcoin at the day of event. There is one interesting result that negative events have higher effect on price of Bitcoin, but it can be caused of importance of events or risk averse of investors. It is difficult to measure importance of events, but from personal view negative events look more important than positive events as it can be seen in history of Bitcoin. It could explain the higher negative effect of negative events. Furthermore, market of cryptocurrencies cannot obtain known market anomalies, because as it was explained above there cannot be weekend effect because Bitcoin is traded 7 days per week. There couldn't be January effect, because cryptocurrencies are not basically used to optimize taxes of investors and expectation of big sold out in December is low. Furthermore, taxation of Bitcoin is complicated, because the main feature of Bitcoin is anonymity (Marian, 2013). Other anomalies of financial market are more likely connected with stock of companies such as underpricing of IPO, low book value and others. We can conclude that markets of cryptocurrencies follow hypothesis of efficiency markets, 
because publicly known information are included in prices and benefit from investment can gain only individuals with insider information.

Additionally, there are needed some improvements of these models as better variables or there could be used better model than ECM that could predict better results.

\section{Conclusion}

Cryptocurrencies are new economic and financial instrument with special and innovative features. Probably the most important one of them is that they have no underlying asset, they are not issued by any government or central authority and they bring no interest or dividends. However, cryptocurrencies are becoming more and more popular and many merchants accept payments, especially online. There is growing market share of Bitcoin. There were rapid boosts and falls that is connected with high price volatility and there is increasing interest among users and academic researches as well.

This paper attempts to analyze the effect of public announcements on price of Bitcoin. The paper analyses the relationship between price of cryptocurrencies and announced positive or negative events. There was used methodology of Error Correction Model on daily data from March 2013 to end of August 2014.

The empirical analyses confirm that prices of cryptocurrencies namely Bitcoin react on publicly announced information and it follows hypothesis of efficient markets. Specifically, there were found that price of Bitcoin is higher during days of positive events and lower during days of negative events than during other days without any events. The detailed analysis confirmed the importance of events on prices of cryptocurrencies. According to the hypothesis of efficient markets, prices of cryptocurrencies reflect all known information and no one could outperform the market by using the same information that is already available to all speculators, except through luck or some insider information.

Secondly, analyses confirm that Bitcoin market determinants have an important effect on Bitcoin price. In the other words, supply and demand factors have crucial impact on the price of Bitcoin and it follows a standard economic model of currency price formation. Particularly, demand side factors are more important, because supply side is given exogenously and it will be fixed in future.

Thirdly, our estimates do not support previous findings that global macroeconomics and financial developments and speculations of investors might drive price of Bitcoin. There weren't any statistically significant impact of Wikipedia search queries and macrofinancial indicators on price of Bitcoin. In fact, there were the highest weaknesses of model, because the relevance of variable could be poor and those proxies could be replaced with more appropriate factors.

This paper serves insights into cryptocurrencies markets and there are many possible improvements that were suggested and cryptocurrencies are still innovative instruments that need to be studied and enhanced. There are limited numbers of researches on 
Litecoin and Dogecoin that need to be done. Therefore, price formation of those instruments is not estimated, because lack of data.

\section{References}

Bakos, Y. (1998). The Emergining Role of Electronic Marketplaces on the Internet. Communications of the ACM .

Bårdsen, G. (1989). Estimation of long run coefficients in error correction models. Oxford Bulletin of Economics and Statistics, pp 345-350.

Brezo, F., \& Bringas, P. G. (2012). Issues and Risks Associated with Cryptocurrencies such as Bitcoin. SOTICS.

Briére, M., Oosterlinck, K., \& Szafarz, A. (2013). Virtual Currency, Tangible Return: Portfolio Diversification with Bitcoins. Centre Emile Bernheim .

Brito, J., Shadab, H., \& Castillo, A. (2014). Bitcoin Financial Regulation: Securities, Derivates, Prediction Markets, \& Gambling.

Buchholz, M., Delaney, J., Warren, J., \& Parker, J. (2012). Bits and Bets: Information, Price Volatility and Demand for Bitcoin.

Chowdbury, A. (2014). Is Bitcoin the "Paris Hilton" of the Currency World? Or Are the Early Investors onto Something That Will Make Them Rich? Marquette University .

Ciaian, P., Rajcaniova, M., \& d'Artis, K. (2014). The Economics of Bitcoin Price Formation.

European Central Bank. (2012). Virtual Currency Schemes. European Central Bank.

Fama, E. F. (1970). Efficient capital markets: A review of theory and empirical work. pp. 383-417.

French, K. (1979). Stock returns and the weekend effect. University of Rochester .

Glaser, F., Zimmermann, K., Haferkorn, M., Weber, M. C., \& Siering, M. (2014). BITCOIN - ASSET OR CURRENCY? REVEALING USERS' HIDDEN INTENTIONS. Twenty Second European Conference on Information Systems .

Griffiths, R., Lim, G. C., \& Hill, C. (2012). Principles of Econometrics. John Wiley \& Sons .

Kristoufek, L. (2013). Bitcoin meets Google Trends and Wikipedia: Quantifying the relationship between phenomena of Internet era. Scientific Reports .

Lerner, J. (2003). The New Financial Thing: The Origins of Financial Innovations. Harvard University and National Bureau of Economic Research .

Maggi, M. (2014). Bitcoin Shop, Inc. (BTCS). MM .

Marian, O. Y. (2013). Are Cryptocurrencies Super Tax Haven? University of Florida Levin College of Law.

Nakamoto, S. (2008). Bitcoin: A Peer-to-Peer Electronic Cash System. www.bitcoin.org

Singal, V. (2006). Beyond the random walk: a guide to stock market anomalies and low risk investic. pp. 47-48.

van Wijk, D. (2013). What can be expected from Bitcoin? Erasmus Universiteit Rotterdam .

Yermack, D. (2013). Is Bitcoin a real currency? An economic appraisal. NATIONAL BUREAU OF ECONOMIC RESEARCH. 